\title{
Histopathology of Women with "Atypical Squamous Cells Cannot Exclude High-Grade Squamous Intraepithelial Lesion" (ASC-H) Smears
}

\author{
Sasivimon Ratree, Pilaiwan Kleebkaow*, Apiwat Aue-Aungkul, Amornrat \\ Temtanakitpaisan, Bandit Chumworathayi, Sanguanchoke Luanratanakorn
}

\begin{abstract}
Objectives: To evaluate prevalence of underlying significant pathologies among women with cervical smears rated as 'atypical squamous cells cannot exclude high grade squamous intraepithelial lesion (ASC-H)', as well as associated risk factors. Methods: Medical records were reviewed of all consecutive women with ASC-H smears who had undergone colposcopy at Srinagarind Hospital from January 2008 to July 2016. Significant pathology results included cervical intraepithelial neoplasia (CIN) 2-3, adenocarcinoma in situ (AIS), endometrial hyperplasia, and cancer of any original site. Result: During the study period, 133 women with ASC-H were reviewed. The mean age was 45.3 years (range 21-72). The histopathologic results for the 133 women were as follows: no lesions $(58 ; 43.6 \%)$, CIN 1 (34; 25.6\%), CIN 2-3 (33; 24.8\%), AIS $(2 ; 1.5 \%)$, and cervical cancer $(6 ; 4.5 \%)$. The overall rate of significant pathology was 30.8\% (95\% confidence interval, $22.9 \%-38.8 \%)$. Women younger than 40 years old carried a higher risk of harboring significant lesions when compared to older women ( $41.7 \%$ versus $27.8 \%$, respectively). There was no significant impact of parity and menopausal status on the risk of significant pathology results. Conclusion: The rate of significant histopathologies among women with ASC-H smears in this study was approximately $31 \%$ and the associated risk factor was patient age.
\end{abstract}

Keywords: Atypical squamous cells- ASC-H- cervical intraepithelial neoplasia- cervical cancer

Asian Pac J Cancer Prev, 20 (3), 683-686

\section{Introduction}

Cervical cytology remains the most common method used for cervical cancer screening. The most common category of cervical epithelial abnormality is atypical squamous cell (ASC) (Solomon et al., 2002). Atypical squamous cells can be subdivided into atypical squamous cells of undetermined significance (ASC-US) and atypical squamous cells cannot exclude high-grade squamous intraepithelial lesion (ASC-H) (Solomon et al., 2002). The latter is defined by abnormal cytological features that suggest high-grade squamous intraepithelial neoplasia (HSIL). However, these features alone are not sufficient for diagnosis (Solomon et al., 2002).

In the 2012 "Updated Consensus Guidelines for the Management of Abnormal Cervical Cancer Screening Tests and Cancer Precursors" proposed by the American Society for Colposcopy and Cervical Pathology (ASCCP), ASC-H is considered to be a high-grade smear abnormality (Massad et al., 2013). However, this should be considered cautiously as there is a low inter-observer agreement for interpreting ASC-H smears due to lax diagnostic criteria.
Therefore, it is no surprise that prevalence rates of underlying significant histopathology of the cervix among women with smears suggesting ASC-H reported in the literature vary widely from $10 \%$ to $80 \%$ (McHale et al., 2007; You et al., 2010; Nogara et al., 2011; Kietpeerakool et al., 2009; Cetiner et al., 2013; Kietpeerakool et al., 2008; Bandyopadhyay et al., 2008; Sung et al., 2011; Lee et al., 2006; Cytryn et al., 2009; Selvaggi, 2013). Smears suggesting ASC-H are extremely rare with a reported rate of less than $0.5 \%$ of all interpretations (You et al., 2010; Cetiner et al., 2013).

Management of abnormal cervical cytology mainly depends on the risk of underlying significant cervical histopathology found in each abnormal smear category. The wide variation in the rates of underlying histopathology among women with ASC-H smears in the literature may lead to questions with regard to the appropriate management in these cases. The purpose of this research is to evaluate the rate of histopathological results indicating high-grade cervical lesions and associated factors in patients with ASC-H smears who presented at the Colposcopy Clinic in Khon Kaen University's Srinagarind 
Hospital. The findings obtained from the present study provide important data for consideration in the treatment of women with ASC-H smears.

\section{Materials and Methods}

In our institute, colposcopies with histological work-ups were carried out in all women with ASC-H smears. After receiving approval of the Research Ethics Committee, medical records were reviewed of women with ASC-H who underwent colposcopic examination and had undergone a histologic work-up within one year following index smears at the Khon Kaen University Colposcopy Clinic from January 2008 to July 2016. Women were excluded if they were pregnant, had previously treated of cancer and a previous history of abnormal cervical cytology or undergone hysterectomy prior to the colposcopic evaluation. Because this was a retrospective study and the data were analyzed and reported anonymously, the need for informed consent was waived by the Research Ethics Committee.

Abstracted data included patient characteristics, detailed colposcopic findings, methods of tissue sampling, and final histopathology results. Colposcopic examination by using the application of $5 \%$ acetic acid solution on the upper vagina and cervix was performed by four gynecologic oncologists. The severity of colposcopic findings was based on severity of acetowhite epitheliums, patterns of abnormal vessels (e.g. punctuation, mosaic), irregular surfaces, inner border signs, ridge signs, and presence of atypical vessels. When multiple biopsies were available, only the most severe diagnosis was recorded. If there was no significant lesion, a follow-up cytology would be performed at 6 months. Significant pathology results included cervical intraepithelial neoplasia (CIN) 2-3, adenocarcinoma in situ (AIS), endometrial

Table 1. Characteristics of 133 Women with ASC-H Smears

\begin{tabular}{lc}
\hline Characteristics & Number (\%) \\
\hline Age (years) & $36(27.1)$ \\
$<40$ & $97(72.9)$ \\
$\geq 40$ & \\
Menopausal status & $103(77.4)$ \\
Premenopause & $30(22.6)$ \\
Postmenopause & \\
Parity status & $14(10.5)$ \\
Nulliparity & $119(89.5)$ \\
Multiparity & \\
Final histopathology results & $58(43.6)$ \\
No lesions & $34(25.6)$ \\
CIN 1 & $33(24.8)$ \\
CIN 2-3 & $2(1.5)$ \\
AIS & $6(4.5)$ \\
Cervical cancer &
\end{tabular}

ASC-H, atypical squamous cells cannot exclude high-grade squamous intraepithelial lesions; CIN, cervical intraepithelial neoplasia; AIS, adenocarcinoma in situ. hyperplasia, and cancer of any original sites. Invasive cancers were staged according to the 2009 revised International Federation of Gynecology and Obstetrics (FIGO) System (Solomon et al., 2002). All surgical specimens were initially reported or reviewed by a Gynecologic Pathologist (PK).

Statistical analysis was performed using SPSS statistic package for Windows. Descriptive statistics were used for reporting demographic data and detailed histology results. A 95\% confidence interval (CI) of the rate of underlying significant lesions was calculated to report the precision of the data. Multivariate analysis using a logistic regression model was used to find independent risk factors for harboring significant lesions.

\section{Results}

During the study period, 133 women with ASC-H were reviewed. Table 1 shows the baseline characteristics of the patients. The mean age was 45.3 years (range $21-72$ years). Fourteen (10.5\%) women were nulliparous. Almost all of the cervical smears in this study were conventionally prepared $(n=130)$. Three women were infected with human immunodeficiency virus (HIV). After colposcopy, 86 women underwent cervical conization and/or hysterectomy.

The histopathologic results of 133 women were as follows: no lesions (58 women; $43.6 \%$ ), CIN 1 (34 women; $25.6 \%$ ), CIN 2-3 (33 women; $24.8 \%$ ), AIS ( 2 women; $1.5 \%$ ), and cervical cancer (6 women; $4.5 \%$ ). The overall rate of results indicating significant pathology was $30.8 \%$ (95\% confidence interval, $22.9 \%-38.8 \%$ ). The FIGO stages of six patients found to have invasive cervical cancer were as follows: stage IA1 (2), stage IB1 (2), stage IIA1 (1) and stage IIB (1).

Table 2 displays an analysis performed to determine the factors associated with results indicating significant underlying pathology. Women younger than 40 years old carried a higher risk of harboring significant lesions when compared to older women $(41.7 \%$ versus $26.8 \%$, respectively; adjusted odds ratio, 2.70; 95\% confidence

Table 2. Histopathology of 133 Women with ASC-H Stratified by Baseline Characteristics

\begin{tabular}{lcc}
\hline Characteristics & $\begin{array}{c}\text { Proportion of } \\
\text { women with } \\
\text { significant lesionsa }\end{array}$ & $\begin{array}{c}\text { Adjusted ORb (95\% } \\
\text { CI) }\end{array}$ \\
\hline $\begin{array}{l}\text { Age (years) } \\
<40(\mathrm{n}=36)\end{array}$ & $15(41.67)$ & $2.70(1.11-6.56)$ \\
$\geq 40(\mathrm{n}=97)$ & $26(26.80)$ & Reference category \\
Menopause status & $29(28.15)$ & $0.39(0.15-1.01)$ \\
$\begin{array}{l}\text { Premenopause }(\mathrm{n}=103) \\
\text { Postmenopause }(\mathrm{n}=30)\end{array}$ & $12(40.0)$ & Reference category \\
Parity & $4(28.57)$ & $0.92(0.27-3.13)$ \\
Nulliparous $(\mathrm{n}=14)$ & $37(31.09)$ & Reference category \\
\hline Multiparous $(\mathrm{n}=119)$ &
\end{tabular}

ASC-H, atypical squamous cells cannot exclude high-grade squamous intraepithelial lesions; OR, odds ratio; CI, confidence interval; a, Including cervical intraepithelial neoplasia 2-3 (33), adenocarcinoma in situ (2), and cervical cancer (6); b, Including patient's age, menopausal status, and parity status in the model. 
Table 3. Rate of Significant Lesions Stratified by Patient Age in Previous Studies

\begin{tabular}{lccc}
\hline Authors & $\begin{array}{c}\text { Year of } \\
\text { publication }\end{array}$ & $\begin{array}{c}\text { Age cut-off } \\
\text { point (years) }\end{array}$ & $\begin{array}{c}\text { Rate of } \\
\text { significant } \\
\text { lesions* }\end{array}$ \\
\hline $\begin{array}{l}\text { Kietpeerakool } \\
\text { et al }\end{array}$ & 2008 & $<40$ & $71.50 \%$ \\
Bandyopadhyay & 2008 & $\geq 40$ & $68.80 \%$ \\
et al & & $\geq 40$ & $21.10 \%$ \\
Sung et al & 2011 & $<40$ & $7.60 \%$ \\
& & $\geq 40$ & $52.30 \%$ \\
This study & 2017 & $<40$ & $41.70 \%$ \\
& & $\geq 40$ & $25.80 \%$ \\
Lee et al & 2006 & $<50$ & $38.80 \%$ \\
& & $\geq 50$ & $30.00 \%$ \\
Cytryn et al & 2009 & $<50$ & $22.20 \%$ \\
& & $\geq 50$ & $8.30 \%$ \\
Selvaggi et al & 2013 & $<55$ & $54.70 \%$ \\
& & $\geq 55$ & $35.00 \%$ \\
\hline
\end{tabular}

interval, 1.11-6.56). There was no significant impact of parity (nulliparity versus multiparity) or menopausal status (premenopause versus postmenopause) on the risk of significant pathology results.

\section{Discussion}

In this study, the authors determined the rate of significant underlying lesions among women with ASC-H cervical smears who had undergone immediate colposcopy, as well as its associated predictors. The rate of significant underlying lesions was $30.8 \%$. The rate of invasive cancer was $4.5 \%$ and all were cervical cancer. The only significant factor predicting significant lesions was the patient age.

As mentioned above, the laxness of the criteria for cytological interpretation of ASC-H leads to a wide variation $(10 \%-80 \%)$ of underlying histopathology results obtained within 12 months following the index smear (McHale et al., 2007; You et al., 2010; Nogara et al., 2011; Kietpeerakool et al., 2009; Cetiner et al., 2013; Kietpeerakool et al., 2008; Bandyopadhyay et al., 2008; Sung et al., 2011; Lee et al., 2006; Cytryn et al., 2009; Selvaggi, 2013). The administration of a human papillomavirus (HPV) test has been proposed as a reflex investigation in order to identify women with a higher risk of significant lesions (Bandyopadhyay et al., 2008; Sung et al., 2011). In a previous study only $1.2 \%$ of women with negative HPV tests had CIN 2-3+, which is significantly lower than the $32.7 \%$ in women with positive HPV tests (Bandyopadhyay et al., 2008). In a study conducted among Korean women, HPV positivity in ASC-H smears was significantly associated with significant underlying lesions, irrespective of patient age. The detection rate of significant lesions was $73.9 \%$ in women with positive HPV tests compared to $18.4 \%$ in women with negative HPV tests (Sung et al., 2011). In the meta-analysis conducted to evaluate the role of HPV tests in for managing women with ASC-H smears, the average risks was $34 \%$ for CIN2+ and $20 \%$ for CIN3 + . A negative HPV test decreased the risk in cases of CIN2+ and CIN $3+$ to just $8 \%$ and $5 \%$, respectively, whereas a positive test increased the risk to $47 \%$ and $28 \%$, respectively (Xu et al., 2016). However, the authors suggest that the utility of an HPV test may be limited if there is a high probability of significant lesions in cases with cytological interpretation of ASC-H (Xu et al., 2016).

Several baseline characteristics have been observed as factors influencing the risk of significant lesions among women with ASC-H including patient age, menopausal status, and body mass index (BMI) (Kietpeerakool et al., 2008; Bandyopadhyay et al., 2008; Sung et al., 2011; Lee et al., 2006; Cytryn et al., 2009; Selvaggi, 2013; Saad et al., 2006; Ahn et al., 2012).

False-positives are more common in the ASC-H smears of older women than in those of younger women. However, the age cut-off point that is applied varies across studies. Table 3 displays the results of previous studies that reported the impact of patient age on the risk of significant lesions among women with ASC-H smears (Kietpeerakool et al., 2008; Bandyopadhyay et al., 2008; Sung et al., 2011; Lee et al., 2006; Cytryn et al., 2009; Selvaggi, 2013). A similar result was also noted in the present study as women younger than 40 years carried a higher risk of harboring significant lesions when compared to older women $(41.7 \%$ versus $26.8 \%$, respectively; Table 2).

A previous study noted that approximately $22 \%$ of premenopausal women who had undergone a histologic follow-up were found to have high-grade histologic lesions compared to only $6 \%$ of postmenopausal women (Saad et al., 2006). The difficulty in distinguishing atrophyrelated epithelial changes from high-grade abnormality has been proposed as an explanation for the high false-positive rate of high-grade smear abnormality (Kietpeerakool et al., 2007).

In a study conducted among postmenopausal Asian women with cervical smears indicating ASC-H, the rate of high-grade lesions among those women who were overweight/obese was higher than that in women who were normal/underweight $(29.8 \%$ versus $18.6 \%$, respectively) (Ahn et al., 2012). The authors of that study postulated that the low levels of estrogen in patients with a lower BMI, could lead to more severe atrophic changes to cervical cells, which mimics high-grade abnormality and may contribute to higher false-positive rates (Ahn et al., 2012).

Although there are several differences in terms of sampling methods and preparation techniques between conventional and liquid-based cervical cytology, previous studies observed no significant impact of smear preparation type on the rate of underlying cervical lesions among women with ASC-H smears (Sung et al., 2011; Ahn et al., 2012; Louro et al., 2003).

Some limitations of this study are worthy of note. Firstly, this study contained a relatively small sample size, which was due to the rarity of ASC-H smears. Secondly, some data were not available, such as previous history of cervical cancer screening, sexual behavior, and BMI, 
which might influence the rate of significant underlying lesions in the current study. Thirdly, a central slide review was not available owing to the impracticality of tracking the specimens in our setting. Finally, we were unable to assess the influences of HIV infection, high-risk HPV infection, and smear preparation techniques on the risk of significant lesions owing to limited data being available.

In conclusion, nearly one-third of the women with ASC-H cervical smears in this study were found to have significant underlying lesions following immediate colposcopy and the associated risk factor for this was patient age. Women younger than 40 years old carried a significantly higher risk of harboring significant lesions than older women, thus suggesting that aggressive work-ups should be conducted in these cases.

\section{Conflict of interest}

The authors have no conflicts of interest.

\section{References}

Ahn S, Lee YY, Sung JY, et al (2012). Body mass index and outcome of ASC-H- interpreted cervical smears in postmenopausal women. Acta Cytol, 56, 259-65.

Bandyopadhyay S, Austin RM, Dabbs D, Zhao C (2008). Adjunctive human cannot exclude high-grade squamous intraepithelial lesion. A follow-up study of conventional and liquid-based preparations in a high-risk population. $\mathrm{Am}$ J Clin Pathol, 120, 392-7.

Cetiner H, Kir G, Kaygusuz E, Saglican Y, Kabaca C (2013). Is the low-grade squamous intraepithelial lesion/atypical squamous cells cannot exclude high-grade squamous intraepithelial lesion category associated with cervical intraepithelial neoplasia 2?. Acta Cytol, 57, 581-4.

Cytryn A, Russomano FB, Camargo MJ, et al (2009). Prevalence of cervical intraepithelial neoplasia grades II/III and cervical cancer in patients with cytological diagnosis of atypical squamous cells when high-grade intraepithelial lesions (ASC-H) cannot be ruled out. Sao Paulo Med J, 127, 283-7.

Kietpeerakool C, Cheewakriangkrai C, Suprasert P, Srisomboon J (2009). Feasibility of the 'see and treat' approach in management of women with 'atypical squamous cell, cannot exclude high-grade squamous intraepithelial lesion' smears. J Obstet Gynaecol Res, 35, 507-13.

Kietpeerakool C, Srisomboon J, Tantipalakorn C, et al (2008). Underlying pathology of women with "atypical squamous cells, cannot exclude high-grade squamous intraepithelial lesion" smears, in a region with a high incidence of cervical cancer. J Obstet Gynaecol Res, 34, 204-9.

Kietpeerakool C, Srisomboon J, Sukkawattananon W, Siriaunkgul S (2007). How can the overtreatment rate of "see and treat" approach be reduced in women with high-grade squamous intraepithelial lesion on cervical cytology?. Asian Pac J Cancer Prev, 8, 206-8.

Lee SJ, Jung KL, Lee JW, et al (2006). Analyses of atypical squamous cells refined by the 2001 Bethesda System: the distribution and clinical significance of follow-up management. Int J Gynecol Cancer, 16, 664-9.

Louro AP, Roberson J, Eltoum I, Chhieng DC (2003). Atypical squamous cells, cannot exclude high-grade squamous intraepithelial lesion. A follow-up study of conventional and liquid-based preparations in a high-risk population. $\mathrm{Am}$ $J$ Clin Pathol, 120, 392-7.

Massad LS, Einstein MH, Huh WK, et al (2013). 2012 updated consensus guidelines for the management of abnormal cervical cancer screening tests and cancer precursors. J Low Genit Tract Dis, 17, 1-27.

McHale MT, Souther J, Elkas JC, Monk BJ, Harrison TA(2007). Is atypical squamous cells that cannot exclude high-grade squamous intraepithelial lesion clinically significant?. J Low Genit Tract Dis, 11, 86-9.

Nogara PR, Manfroni LA, Consolaro ME (2011). Cervical cytology of atypical squamous cells cannot exclude high-grade squamous intraepithelial lesion (ASC-H): histological results and recurrence after a loop electrosurgical excision procedure. Arch Gynecol Obstet, 284, 965-71.

Pecorelli S (2009). Revised FIGO staging for carcinoma of the vulva, cervix, and endometrium. Int J Gynaecol Obstet, 105, 103-4.

Saad RS, Dabbs DJ, Kordunsky L, et al (2006). Clinical significance of cytologic diagnosis of atypical squamous cells, cannot exclude high grade, in perimenopausal and postmenopausal women. Am J Clin Pathol, 126, 381-8.

Selvaggi SM (2013). Clinical significance of atypical squamous cells cannot exclude high grade squamous intraepithelial lesion with histologic correlation-: a 9-year experience. Diagn Cytopathol, 41, 943-6.

Solomon D, Davey D, Kurman R, et al (2002). The 2001 Bethesda System: terminology for reporting results of cervical cytology. JAMA, 287, 2114-9.

Sung CO, Oh YL, Song SY (2011). Cervical cytology of atypical squamous cells, cannot exclude high-grade squamous intraepithelial lesion: significance of age, human papillomavirus DNA detection and previous abnormal cytology on followup outcomes. Eur J Obstet Gynecol Reprod Biol, 159, 155-9.

Xu L, Verdoodt F, Wentzensen N, Bergeron C, Arbyn M (2016). Triage of ASC-H: A meta-analysis of the accuracy of high-risk HPV testing and other markers to detect cervical precancer. Cancer Cytopathol, 124, 261-72.

You K, Guo Y, Gen L, Qiao J (2010). The risk of CIN II or greater in a one-year follow-up period in patients with ASC-H interpreted with cytology. Eur J Obstet Gynecol Reprod Biol, 149, 215-7.

\section{(ब) $(\mathbb{2} \otimes$}

This work is licensed under a Creative Commons AttributionNon Commercial 4.0 International License. 